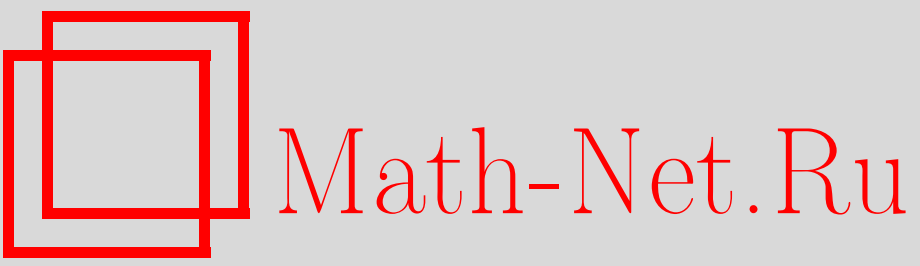

В. И. Матвеев, Приближение внезапных возмущений для уравнения Дирака, TMФ, 2005, том 142, номер 1, 58-63

DOI: https://doi.org/10.4213/tmf1772

Использование Общероссийского математического портала Math-Net.Ru подразумевает, что вы прочитали и согласны с пользовательским соглашением

http://www.mathnet.ru/rus/agreement

Параметры загрузки:

IP: 54.237 .59 .107

26 апреля 2023 г., 16:49:41 
ТЕОРЕТИЧЕСКАЯ

И МАТЕМАТИЧЕСКАЯ

ФИЗИКА

Том 142, № 1

январь, 2005

(C) 2005 г.

В. И. Матвеев*

\section{ПРИБЛИЖЕНИЕ ВНЕЗАПНЫХ ВОЗМУШЕНИЙ ДЛЯ УРАВНЕНИЯ ДИРАКА}

В приближении внезапных возмущений получено решение уравнения Дирака, описывающее поведение водородоподобного атома при взаимодействии с пространственно неоднородным ультракоротким импульсом электромагнитного поля. Соответствующие вероятности переходов выражены через известные неупругие атомные формффакторы, широко используемые в теории релятивистских столкновений заряженных частиц с атомами.

Ключевые слова: атом, ультракороткий импульс электромагнитного поля, приближение внезапных возмущений, уравнение Дирака.

\section{1. ВВЕДЕНИЕ}

Приближение внезапных возмушений [1], [2] в нерелятивистской квантовой механике является основой расчетных методик в тех случаях, когда возмушение не является достаточно малым для применения теории возмущений, однако время действия возмушения значительно меньше характерных периодов времени невозмушенной системы. Это позволяет решать задачу, не ограничивая величину возмушения. Известно много примеров, когда происходит возбуждение или ионизация атома под действием внезапного возмушения. Прежде всего, это возбуждение или ионизация атомов при ядерных реакциях [2], [3], например при $\beta$-распаде ядра, когда вылет быстрого $\beta$-электрона воспринимается атомными электронами как внезапное изменение заряда ядра, или при ударе нейтрона о ядро, когда происходит внезапная передача импульса ядру, и т.п. Приближение внезапных возмушений применяется при рассмотрении многоэлектронных переходов в сложных атомах, когда переходы, происходящие во внутренних оболочках, воспринимаются сравнительно медленными электронами внешних оболочек как мгновенные [4]. Как результат действия внезапного возмущения рассматриваются неупругие процессы при столкновениях быстрых многозарядных ионов с атомами [5], [6] и при столкновениях заряженных частиц с высоковозбужденными атомами [7]. К подобным случаям можно также отнести эффекты взаимодействия атомов с ультракороткими импульсами электромагнитного поля, которые в настояшее время становятся объектами экс-

* Поморский государственный университет, Архангельск, Россия. E-mail: matveev.victor@pomorsu.ru 
периментальных и теоретических исследований. Под ультракороткими импульсами в рамках данной статьи понимаются импульсы длительностью, меньшей характерных периодов времени для атома-мишени, который до взаимодействия может находиться как в основном, так и в возбужденном, в том числе в высоковозбужденном (ридберговском) состоянии. Такие импульсы могут иметь различное происхождение [8], в частности, они могут быть полями движущихся с релятивистской или ультрарелятивистской скоростью тяжелых ионов [9], причем в последнем случае для полей ионов с достаточно большими зарядами теория возмушений неприменима даже при сколь угодно больших энергиях ионов [5], [10]. В работе [11] на основе приближения внезапных возмушений была развита нерелятивистская теория, описывающая электронные переходы и излучение атома при его взаимодействии с пространственно неоднородным ультракоротким импульсом электромагнитного поля, получены вероятности возбуждения и ионизации, а также спектры и сечения переизлучения атомом такого импульса. Развитая в [11] методика позволяет произвести точный учет как пространственной неоднородности (на размерах атома-мишени) поля ультракороткого импульса, так и импульсов фотонов в процессах переизлучения.

В настоящей работе на основе приближения внезапных возмушений получено решение уравнения Дирака, описывающее поведение водородоподобного атома при взаимодействии с пространственно неоднородным ультракоротким импульсом электромагнитного поля. Соответствующие вероятности переходов выражены через известные неупругие атомные формфакторы, широко используемые в теории релятивистских столкновений заряженных частиц с атомами.

\section{2. АМПЛИТУДА ПЕРЕХОДА В ПРИБЛИЖЕНИИ ВНЕЗАПНЫХ ВОЗМУШЕНИЙ}

По терминологии [1] возмущение, соответствуюшее полю ультракороткого импульса, имеет характер встряски типа рассеяния. Для иллюстрации приближения внезапных возмущений в этом случае, видимо, проще всего рассмотреть формальное решение уравнения Шредингера (здесь и далее используются атомные единищы)

$$
i \dot{\Psi}=\left(H_{0}+U(t)\right) \Psi
$$

где внезапное возмущение $U(t)$ действует в течение времени, значительно меньшего, чем характерные периоды времени невозмущенной системы, описываемой гамильтонианом $H_{0}$. Тогда при решении уравнения (1) можно (в течение времени действия возмушения $U(t))$ пренебречь эволюцией волновой функции под действием собственного гамильтониана $H_{0}$ и решать уравнение $i \dot{\Psi}=U(t) \Psi$. Отсюда следует, что

$$
\Psi(t)=\exp \left\{-i \int_{t_{0}}^{t} U(t) d t\right\} \Psi\left(t_{0}\right) .
$$

Поэтому амплитуда перехода нерелятивистского атома из состояния $|f\rangle$ в состояние $|j\rangle$ в результате внезапного возмушения $U(t)$ имеет вид [1]

$$
a_{f j}=\left\langle j\left|\exp \left\{-i \int_{-\infty}^{+\infty} U(t) d t\right\}\right| f\right\rangle .
$$


Легко видеть, что тот же результат для амплитуды перехода может быть получен, если точно решить уравнение $(1)$ с дельтаобразным потенциалом $\widetilde{U}(t)$, связанным с потенциалом $U(t)$ следуюшим образом:

$$
\widetilde{U}(t)=U_{0} \delta(t), \quad U_{0}=\int_{-\infty}^{+\infty} U(t) d t .
$$

Именно это обстоятельство мы используем ниже для решения уравнения Дирака в приближении внезапных возмущений.

Поведение электрона водородоподобного атома (с зарядом ядра $Z_{a}$, на значения которого мы не будем накладывать ограничений, кроме условий применимости [12] уравнения Дирака) во внешнем поле $\mathbf{A}, \varphi$ будем описывать уравнением Дирака (заряд электрона равен -1 ат.ед.)

$$
i \dot{\Psi}=\left\{c \boldsymbol{\alpha}\left(\hat{\mathbf{p}}+\frac{1}{c} \mathbf{A}\right)-\frac{Z_{a}}{r}-\varphi-\beta c^{2}\right\} \Psi,
$$

где члены $c \boldsymbol{\alpha} \hat{\mathbf{p}}-\beta c^{2}-Z_{a} / r$ представляют собой гамильтониан $H_{0}$ изолированного атома, взаимодействие атомного электрона с внешним полем равно $U(t)=U(\mathbf{r}, t)=\boldsymbol{\alpha} \mathbf{A}-$ $\varphi ; \hat{\mathbf{p}}$ - оператор импульса, $\boldsymbol{\alpha}$ и $\beta$ - матрицы Дирака, $c$ - скорость света, $\mathbf{r}-$ координаты атомного электрона. Сначала выберем калибровку векторного и скалярного потенциалов электромагнитных волн так, что скалярный потенщиал $\varphi$ равен нулю. Будем считать, что векторный потенциал $\mathbf{A}$ поля волны зависит от координат $\mathbf{r}$ и времени $t$ следующим образом: $\mathbf{A}(\mathbf{r}, t)=\mathbf{A}(\eta)$, где фаза волны $\eta=\omega_{0} t-\mathbf{k}_{0} \mathbf{r}$, волновой вектор $\mathbf{k}_{0}$ таков, что $\left|\mathbf{k}_{0}\right|=\omega_{0} / c, \omega_{0}-$ круговая частота. Проведем калибровочное преобразование [11]:

$$
\mathbf{A}^{\prime}=\mathbf{A}+\nabla f, \quad \varphi^{\prime}=\varphi-\frac{1}{c} \frac{\partial f}{\partial t}, \quad f=\mathbf{A} \mathbf{r} .
$$

В результате получаем $\mathbf{A}^{\prime}=-\mathbf{k}_{0}(\mathbf{r} d \mathbf{A} / d \eta), \varphi^{\prime}=-(\mathbf{E r})$, где $\mathbf{E}=\mathbf{E}(\mathbf{r}, t)=-\left|\mathbf{k}_{0}\right| \times$ $d \mathbf{A} / d \eta$. Поэтому в новой калибровке векторный и скалярный потенциалы связаны следующим образом: $\mathbf{A}^{\prime}=\left(\mathbf{k}_{0} /\left|\mathbf{k}_{0}\right|\right) \varphi^{\prime}$. Будем считать ось $Z$ направленной вдоль вектора $\mathbf{k}_{0}$. В этом случае в уравнении (5) взаимодействие атомного электрона с внешним полем равно

$$
U(t)=\boldsymbol{\alpha} \mathbf{A}^{\prime}-\varphi^{\prime}=-\left(1-\frac{\boldsymbol{\alpha} \mathbf{k}_{0}}{\left|\mathbf{k}_{0}\right|}\right) \varphi^{\prime}=-\left(1-\alpha_{z}\right) \varphi^{\prime} .
$$

Далее будем работать в новой калибровке и опускать штрихи у потенциалов. Для решения в приближении внезапных возмушений уравнения Дирака (5) запишем его в виде $i \dot{\Psi}=\left(H_{0}+U(t)\right) \Psi$ и воспользуемся введенной в начале данного раздела заменой $(4)$. Для этого введем

$$
\widetilde{\varphi}=-\varphi_{0} \delta(c t-z), \quad \varphi_{0}=c \int_{-\infty}^{+\infty} \varphi(c t-z) d t .
$$

Соответственно в формуле (6) заменим $U(t)$ на

$$
\widetilde{U}(t)=-\left(1-\alpha_{z}\right) \widetilde{\varphi}=U_{0} \delta(c t-z),
$$


где

$$
U_{0}=c \int_{-\infty}^{+\infty} U(t) d t=-\left(1-\alpha_{z}\right) \varphi_{0} .
$$

В результате уравнение (5) примет вид

$$
i \dot{\Psi}=\left\{c \boldsymbol{\alpha} \hat{\mathbf{p}}-\frac{Z_{a}}{r}-\beta c^{2}-\left(1-\alpha_{z}\right) \widetilde{\varphi}\right\} \Psi
$$

Для получения точного решения уравнения Дирака с таким потеншиалом разложим $\Psi=$ $\Psi(\mathbf{r}, t)$ по собственным функциям $\phi_{k}(\mathbf{r})$ (с энергиями $E_{k}$ ) невозмушенного атомного гамильтониана $H_{0}=c \boldsymbol{\alpha} \hat{\mathbf{p}}-\beta c^{2}-Z_{a} / r$ :

$$
\Psi(\mathbf{r}, t)=\sum_{k} a_{k}(t) \phi_{k}(\mathbf{r}) e^{-i E_{k} t} .
$$

Подставляя это разложение в левую часть уравнения $i \dot{\Psi}=\left(H_{0}+\widetilde{U}(t)\right) \Psi$ и интегрируя, с учетом ортогональности состояний $\phi_{k}$ получаем после умножения слева на какое-либо состояние $\phi_{f}$

$$
\frac{d a_{f}(t)}{d t}=-i e^{i E_{f} t}\left\langle\phi_{f}|\widetilde{U}(t)| \Psi(\mathbf{r}, t)\right\rangle .
$$

Пусть до столкновения атом находился в состоянии $\phi_{j}$, тогда

$$
\begin{aligned}
\left.\Psi(\mathbf{r}, t)\right|_{t=-\infty} & =e^{-i E_{j} t} \phi_{j}(r), \\
\left.a_{f}(t)\right|_{t=-\infty} & =\delta_{f j},
\end{aligned}
$$

где $\delta_{f j}$ - символ Кронекера. Поскольку $\widetilde{U}(t)=U_{0} \delta(c t-z)$, то для решения уравнения (11) достаточно знать значения $\Psi(\mathbf{r}, t)$ лишь при $z=c t$, которые можно найти из уравнения (10) следуюшим образом. Переходим к переменным светового конуса

$$
z^{-}=(c t-z), \quad z^{+}=(c t+z)
$$

и, сохраняя в малой окрестности $z^{-}=0$ только производные по $z^{-}$и сингулярный потенциал $-\left(1-\alpha_{z}\right) \widetilde{\varphi}$, получаем уравнение

$$
i c\left(1-\alpha_{z}\right) \frac{\partial \Psi}{\partial z^{-}}=-\left(1-\alpha_{z}\right) \widetilde{\varphi} \Psi .
$$

Поскольку $\widetilde{\varphi}=-\varphi_{0} \delta\left(z^{-}\right)$, то учитывая соотношения

$$
\frac{d}{d x} \theta(x)=\delta(x), \quad \frac{d}{d x} e^{\theta(x)}=\delta(x) e^{\theta(x)},
$$

где $\theta(x)=0$ при $x<0, \theta(x)=1$ при $x>0$, находим решение уравнения (15)

$$
\left(1-\alpha_{z}\right) \Psi\left(z^{-}+0\right)=\left(1-\alpha_{z}\right) e^{-i \varphi_{0} \theta\left(z^{-}\right) / c} \Psi\left(z^{-}-0\right) .
$$


Отсюда, возврашаясь к времени $t$ и используя условие $(12)$, находим решение при $t<z / c$ и вблизи $t=z / c$ (т.е. при $t=z / c+\varepsilon$, где $\varepsilon>0$ мало):

$$
\left(1-\alpha_{z}\right) \Psi(\mathbf{r}, t)=\left(1-\alpha_{z}\right) e^{-i \theta(c t-z) \varphi_{0} / c} e^{-i E_{j} t} \phi_{j}(\mathbf{r}) .
$$

Подставляя последнее равенство в правую часть (11) и интегрируя по $t$ с учетом начальных условий (13), получаем

$$
\begin{aligned}
a_{f j}= & \left.a_{f}(t)\right|_{t=+\infty}=\delta_{f j}-i \int_{-\infty}^{+\infty} d t e^{i\left(E_{f}-E_{j}\right) t} \times \\
& \times\left\langle\phi_{f}\left|\delta(z-c t)\left(1-\alpha_{z}\right) e^{-i \theta(c t-z) \varphi_{0} / c}\right| \phi_{j}\right\rangle .
\end{aligned}
$$

Используя соотношения (16), находим

$$
a_{f j}=\delta_{f j}+\left\langle\phi_{f}\left|\left(1-\alpha_{z}\right) e^{i\left(E_{f}-E_{j}\right) z / c}\left(e^{-i \varphi_{0} / c}-1\right)\right| \phi_{j}\right\rangle
$$

- искомое точное решение уравнения Дирака с потенциалом $\widetilde{U}(t)=U_{0} \delta(c t-z)$, соответствующее приближению внезапных возмущений для потенциала (6). Полученное выражение можно переписать в более удобном виде, если воспользоваться легко проверяемым соотношением

$$
\left\langle\phi_{f}\left|\left(1-\alpha_{z}\right) e^{i\left(E_{f}-E_{j}\right) z / c}\right| \phi_{j}\right\rangle=\delta_{f j}
$$

В результате имеем

$$
a_{f j}=\left\langle\phi_{f}\left|\left(1-\alpha_{z}\right) e^{i\left(E_{f}-E_{j}\right) z / c} e^{-i \varphi_{0} / c}\right| \phi_{j}\right\rangle
$$

\section{3. ВЕРОЯТНОСТИ ПЕРЕХОДОВ}

Рассмотрим взаимодействие атомного электрона с импульсом электромагнитного поля гауссовой формы (эффективной длительности $\sim \lambda^{-1}$ )

$$
\mathbf{E}(\mathbf{r}, t)=\mathbf{E}_{0} e^{-\lambda^{2}\left(t-\mathbf{k}_{0} \mathbf{r} / \omega_{0}\right)^{2}} \cos \left(\omega_{0} t-\mathbf{k}_{0} \mathbf{r}\right), \quad \varphi=\mathbf{r E}(\mathbf{r}, t), \quad \varphi_{0}=-c \mathbf{q} \mathbf{r}
$$

где

$$
\mathbf{q}=\int_{-\infty}^{+\infty} d t \mathbf{E}(\mathbf{r}, t)=\mathbf{E}_{0} \frac{\sqrt{\pi}}{\lambda} e^{-\omega_{0}^{2} /\left(4 \lambda^{2}\right)}
$$

Вспоминая, что $\alpha_{z}=\boldsymbol{\alpha} \mathbf{k}_{0} / k_{0}$, и вводя вектор $\mathbf{Q}=\left(Q_{x}, Q_{y}, Q_{z}\right)=\left(q, 0, \Omega_{f j} / c\right)$, где $\Omega_{f j}=E_{f}-E_{j}$, перепишем $a_{f j}$ из $(22)$ в виде

$$
a_{f j}=\left\langle\phi_{f}\left|\left(1-\frac{\boldsymbol{\alpha} \mathbf{k}_{0}}{k_{0}}\right) e^{i \mathbf{Q r}}\right| \phi_{j}\right\rangle \text {. }
$$

Направляя ось $Z$ вдоль вектора $\mathbf{Q}$, перепишем (22) в виде

$$
a_{f j}=\left\langle\phi_{f}\left|\left(1-\alpha_{z} \cos \theta\right) e^{i Q z}\right| \phi_{j}\right\rangle-\left\langle\phi_{f}\left|\left(1-\alpha_{x} \sin \theta\right) e^{i Q z}\right| \phi_{j}\right\rangle
$$


где

$$
Q=\sqrt{q^{2}+\frac{\Omega_{f j}^{2}}{c^{2}}}, \quad \cos \theta=\frac{\Omega_{f j}}{\sqrt{c^{2} q^{2}+\Omega_{f j}^{2}}}, \quad \sin \theta=\frac{q}{\sqrt{q^{2}+\Omega_{f j}^{2} / c^{2}}} .
$$

Таким образом, если до взаимодействия (т.е. при $t=-\infty)$ с полем ультракороткого импульса атом находился в состоянии $\phi_{j}$ с энергией $E_{j}$, то вероятность обнаружить после взаимодействия (т.е. при $t=+\infty)$ атом в состоянии $\phi_{f}$ с энергией $E_{f}$ равна

$$
\left|a_{f j}\right|^{2}=\frac{q^{2}}{Q^{2}}\left(\frac{q^{2}}{Q^{2}}\left|F^{f j}\right|^{2}+\left|G_{x}^{f j}\right|^{2}\right) .
$$

Здесь мы, следуя [13], [14], ввели хорошо известные неупругие атомные формфакторы:

$$
F^{f j}=\left\langle\phi_{f}\left|e^{i Q z}\right| \phi_{j}\right\rangle=\frac{Q c}{\Omega_{f j}}\left\langle\phi_{f}\left|\alpha_{z} e^{i Q z}\right| \phi_{j}\right\rangle, \quad G_{x}^{f j}=\left\langle\phi_{f}\left|\alpha_{x} e^{i Q z}\right| \phi_{j}\right\rangle,
$$

широко используемые в теории релятивистских столкновений заряженных частиц с атомами. Поэтому приведенные формулы позволяют [14] рассчитать вероятности возбуждения, ионизации и рождения пар при взаимодействии атома с ультракоротким импульсом электромагнитного поля.

Благодарности. Автор благодарит Российский фонд фундаментальных исследований (грант № 04-02-16177-а) и Фонд ИНTAC (грант INTAS-GSI 03-54-4294) за финансовую поддержку работы.

\section{Список литературы}

[1] А. М. Дыхне, Г. Л. Юдин. УФН. 1978. Т. 125. С. 377.

[2] А. Б. Мигдал. Качественные методы в квантовой теории. М.: Наука, 1975.

[3] Л. Д. Ландау, Е. М. Лифииц. Квантовая механика. М.: Наука, 1989.

[4] В.И. Матвеев, Э.С. Парилис. УФН. 1982. Т. 138. С. 583.

[5] J. Eichler. Phys. Rev. A. 1997. V. 15. P. 1856.

[6] Г. Л. Юдин. ЖЭТФ. 1981. Т. 80. С. 1026; В. И. Матвеев. ЭЧАЯ. 1995. Т. 26. С. 780.

[7] И. С. Персиваль. Столкновение заряженых частиц с высоковозбужденными атомами. В сб.: Атомы в астрофизике. Ред. Ф. Г. Берк, В.Б. Эйснер, Д. Г. Хаммер, И. С. Персиваль. М.: Мир, 1986. C. 87 .

[8] S. E. Harris, A. V. Sokolov. Phys. Rev. Lett. 1999. V. 81. P. 2894; I. P. Christov, M. M. Murnane, H.C. Kapteyn. Opt. Commun. 1998. V. 148. P. 75; A. V. Sokolov, D. D. Yavuz, S. E. Harris. Opt. Lett. 1999. V. 24. P. 557; A.E. Kaplan, P. L. Shkolnikov. Phys. Rev. Lett. 2002. V. 88. P. 074801.

[9] A. J. Baltz. Phys. Rev. Lett. 1997. V. 78. P. 1231; R. Moshammer, W. Schmitt, J. Ullrich et al.. Phys. Rev. Lett. 1997. V. 79. P. 3621; A. V. Selin, A. M. Ermolaev, C. J. Joachain. Phys. Rev. A. 2003. V. 67. P. 012709; A. B. Voitkiv, B. Najjari, J. Ullrich. J. Phys. B. 2003. V. 36. P. 2325.

[10] J. Eichler. Phys. Rep. 1990. V. 193. P. 165.

[11] В. И. Матвеев. ЖЭТФ. 2003. Т. 124. С. 1023.

[12] В. Б. Берестецкий, Е. М. Лифииц, Л. П. Питаевский. Квантовая электродинамика. М.: Наука, 1989.

[13] R. Anholt. Phys. Rev. A. 1979. V. 19. P. 1004.

[14] J. Eichler, W. E. Meyrhoft. Relativistic Atomic Collisions. New York: Academic Press, 1995. 